\title{
Electromyographical Comparison of a Traditional, Suspension Device, and Towel Pull-Up
}

\author{
by \\ Ronald L. Snarr'1, Ashleigh V. Hallmark', Jason C. Casey ${ }^{3}$, Michael R. Esco ${ }^{2}$
}

\begin{abstract}
Strengthening muscles of the back may have various implications for improving functions of daily living, aiding in the transfer of power in throwing, and assist in injury prevention of the shoulder complex. While several versions of the pull-up exist, there is currently no literature comparing their differences. The purpose of this investigation was to compare the electromyographical activity of the latissimus dorsi, posterior deltoid, middle trapezius, and biceps brachii while performing three variations of the pull-up. Resistance-trained men and women $(n=15$, age $=24.87 \pm 6.52$ years $)$ participated in this study by performing traditional pull-ups, suspension device pull-ups, and towel pull-ups in a randomized fashion. Each pull-up was performed for three repetitions with a 1.5 biacromial grip-width for each participant. Normalized (\%MVC) electromyographical values were recorded for each muscle group during each pull-up variation. No significant differences existed within the latissimus dorsi, biceps brachii or posterior deltoid between any of the exercises. For the middle trapezius, towel pull-ups provided significantly lower muscle activity than the traditional pull-up, while no differences between suspension pull-ups and the other variations occurred. In conclusion, only one muscular difference existed between the exercise variations and all versions examined provided electromyographical values, determined by current literature, to invoke a sufficient stimulus to promote increases in muscle strength and hypertrophy. Although further research is needed, practitioners can be confident when programming any of the movement variations examined when attempting to elicit adaptations of muscular strength and hypertrophy.
\end{abstract}

Key words: strength training, suspension training, EMG, resistance training.

\section{Introduction}

The pull-up (PU) is a multi-joint exercise involving movement of the shoulder girdle and elbow joints. This closed kinetic chain exercise is designed to increase muscular strength and endurance of both the upper extremities and torso (Ronai and Scibek, 2014; Youdas et al., 2010). Although most activities of daily living or sports do not require an overhead pulling movement, strengthening the supporting musculature of the shoulder girdle may enhance an individuals' ability to transfer power between the upper and lower extremities during total body movements (Harrison et al., 2011; Willardson, 2004). In addition, since the glenohumeral provides the greatest range of motion compared to all other joints of the body, exercises such as the pull-up are important for increasing its strength and stability.

Most research pertaining to electromyographic (EMG) activity of the major muscles involved during pulling movements of the upper torso entail examining modifications in hand-grip or grip-width (Lehman et al., 2004; Lusk et al., 2010; Signorile et al., 2002; Sperandei et al., 2009; Youdas et al., 2010), but not exercise variation. For example, Youdas et al. (2010) determined that no significant differences existed in the primary movers (i.e., latissimus dorsi, biceps brachii, or trapezius) between a traditional pull-up and chin-up with hands supinated. However, Lusk et al. (2010) observed significant differences in the latissimus dorsi (LD) during

1 - College of Health and Kinesiology, Georgia Southern University, Statesboro, GA USA;

2 - Department of Kinesiology, The University of Alabama, Tuscaloosa, AL USA;

3 - Department of Exercise Science, LaGrange College, LaGrange, GA USA 
hand-grip variations while performing the lat pull-down exercise. It was determined that, irrespective of grip-width (i.e., wide versus narrow), a pronated grip provided significantly greater activation of the LD compared to a supinated grip. While the majority of research focuses on the lat pull-down movement or row, there is a great focus in strength and conditioning programs on pull-up exercise variations to strengthen the shoulder joint.

Several types of the traditional pull-up exist that involve the use of a hanging apparatus, such as a stability device or a pair of towels. Suspension pull-ups (SP) are a variation performed with the use of a suspension device, which is a mode of instability training that employs two independent, freely moving handles. The handles are suspended by two straps with a fixed anchor position above the exerciser (e.g., pull-up bar, smith machine, or ceiling). Suspension devices are typically used with the intent to provide an unstable surface, thereby creating a greater muscular demand when performing typical bodyweight exercises (McGill et al., 2014; Snarr and Esco, 2013).

Towel pull-ups (TP) involve grasping a towel in each hand while performing the pull-up. Anecdotally, the addition of the towels increases the intensity of the exercise by requiring the individual to utilize additional grip strength. A number of subjective claims suggest that the SP and TP provide an advanced, more challenging variation compared to the PU. However, there is no scientific evidence to substantiate or refute such statements. Therefore, research is needed to determine the extent of muscular activation of the primary movers during these types of movements. The purpose of this investigation was to compare the EMG activity of the latissimus dorsi (LD), posterior deltoid (PD), middle trapezius (MT), and biceps brachii (BB) between the PU, SP, and TP. Based upon previous research with suspension training and pull-up devices, it was hypothesized that no differences would exist between any pull-up variations tested since all would require the same joint motions, but only vary in grip conditions.

\section{Methods}

A repeated measures study was performed to determine the differences in muscular demands between common PU variations. Fifteen apparently healthy subjects completed three versions of a pull-up during one laboratory visit. Surface electrodes were used to record muscular activity in microVolts, while subjects performed three repetitions of each pullup (i.e., traditional pull-up (PU), suspension device pull-up (SP), and towel pull-up (TP)). The dependent variable in this study was EMG values obtained for the LD, PD, MT, and BB. The independent variables were the three variations of the pull-up (i.e., $\mathrm{PU}, \mathrm{SP}$, and TP).

\section{Participants}

All subjects were recruited via flyers and word of mouth. Fifteen men $(n=13$; age $=25.15 \pm$ 6.95 yrs; body height $=175.85 \pm 8.04 \mathrm{~cm}$; body mass $=80.42 \pm 10.40 \mathrm{~kg})$ and women $(\mathrm{n}=2$; age $=$ $23 \pm 2.83$ yrs; body height $=163.83 \pm 1.80 \mathrm{~cm}$; body mass $=61.35 \pm 6.44 \mathrm{~kg}$ ) volunteered to participate in this investigation and provided informed consent. In order to take part in the study, subjects were required to complete 5 continuous bodyweight pull-up repetitions with proper form. Subjects were also familiarized with all pull-up variations during recruitment, which were examined prior to data collection. The subjects were free from cardiorespiratory, musculoskeletal, or neurological disorders, as determined by a health and medical questionnaire, and presented no prior injuries that would otherwise affect participating in the study. The investigation was approved by the University's Institutional Review Board at the University of Alabama.

\section{Measures}

\section{Electromyography}

All EMG values were collected using a BIOPAC MP150 BioNomadix Wireless Physiology Monitoring System. Signals were converted from analog to digital with a sampling rate of $2 \mathrm{kHz}$ along with a bandpass filter using a 20 to $400 \mathrm{~Hz}$ cutoff frequency and a fourth-order Butterworth filter. Data was analyzed using Acqknowledge 4.2 software (BIOPAC System, Inc., Goleta, CA). All EMG values were collected as the root mean square value of the raw signal. Subjects' skin sites were prepped for application through shaving, exfoliation, and alcohol cleansing prior to placing electrodes (BIOPAC EL504 disposable Ag- $\mathrm{AgCl}$ ), in order to reduce impedance from dead surface tissue and oils. All electrodes were placed on the 
right side of the body which is consistent with previous literature (Cram and Kasman, 1998).

Electrodes for the LD were placed $4 \mathrm{~cm}$ beneath the inferior angle of the scapula, half the distance between the lateral border of the torso and the spine, spaced $2 \mathrm{~cm}$ apart and at an oblique angle. Electrodes for the PD were placed 2 $\mathrm{cm}$ below the lateral border of the spine of the scapula, $2 \mathrm{~cm}$ apart and angled toward the deltoid tuberosity. Electrodes for the MT were placed 2 $\mathrm{cm}$ apart and parallel to the muscle fibers between the thoracic vertebrae and the medial border of the spine of the scapula. Electrodes for the BB were placed vertically $2 \mathrm{~cm}$ apart directly over the muscle belly on the anterior aspect of the arm. Ground electrodes were placed over the surface of the mid-clavicle.

\section{Procedures}

\section{Exercise Trials}

All data was collected during one visit for each subject. Subjects performed three pull-up variations in addition to maximum voluntary contractions (MVCs) for each muscle group examined. Following the placement of electrodes, MVCs were determined to normalize all EMG signals for testing. Each MVC was performed three times per each muscle group for 6 seconds a piece. The middle two seconds of each contraction were then averaged to obtain the reference value (i.e., MVC). A rest period of $2 \mathrm{~min}$ was allowed between each MVC trial. The MVC collection methods used in this study were consistent with previous research (Konrad, 2005).

- LD: Obtained through isometric replication of a lat pull-down with arms placed in a lateral or frontal position at 90 degrees of flexion.

- PD: Obtained through a secured back, seated position with arms fully extended and angled slightly in front of the subject. Matched resistance was then applied as the subject attempted to extend the arm at the glenohumeral joint.

- MT: Subjects lied prone on an athletic table with the right arm hanging down off the side. Matched resistance was then applied as the subject attempted to horizontally abduct the arm.

- BB: Subjects assumed a kneeling position with the upper arm placed on a bench at 90 degrees. Matched resistance was then applied as subjects attempted to flex the elbow.
After completion of MVCs, subjects were given a $5 \mathrm{~min}$ rest period, after which the three pull-up movements were performed. The exercises were performed in randomized order to prevent data fatigue error. Exercise technique was also inspected before and throughout data collection by a Certified Strength and Conditioning Specialist (e.g., NSCA-CSCS). Each variation of the pull-up was performed for three repetitions. If during the repetition the subject improperly performed the movement (e.g., used momentum by kicking the legs, did not reach the proper height or full depth, etc.), the trial was disregarded and repeated. Each repetition was set at a pace of 4 seconds using a metronome; 2 seconds for both the concentric and eccentric phases. While all subjects were familiar and able to perform each exercise, individuals were not given specific cues during the exercises. Previous research has shown that specific instruction and cueing during a lat pull-down increased LD activation (Snyder and Leech, 2009); thus, cueing was not provided to avoid any changes in muscle activity. During data collection, each subject was allowed a 5-min rest period between exercises to prevent fatigue. The technique for each pull-up that was performed in this study was as follows:

- $\quad P U$ (FIGURE 1): Subjects placed hands in a pronated position at a distance of 1.5 times their bi-acromial width on a secure, overhead pull-up bar and pulled their body up to the bar by adducting at the shoulder joint and flexing at the elbows. Once the bottom of the subjects' chin reached the level of the bar, they slowly returned to the starting position.

- TP (FIGURE 2): Prior to this movement, two cotton towels were placed over the bar at a width of 1.5 times the bi-acromial distance for each subject. Subjects then grasped a draped towel in each hand, with palms facing forward, and performed a pull-up using the same technique as in PU. However, once the subjects' chin reached the level of the hands (instead of the bar), they returned to the starting position.

- $\quad$ SP (FIGURE 3): Prior to testing, a suspension device was secured overhead to the pull-up bar. The suspension straps were placed at the same distance as the towels (i.e., 1.5 times biacromial width). Subjects then completed the pull-ups using the same techniques as 
described above.

Post-data collection, a subjective assessment of the exercises was taken to determine a ranking of the most difficult to least difficult movement.

\section{Statistical Analysis}

Muscle activity during the exercises was recorded in root mean square of the raw signal. The mean activity for each movement across the three repetitions was then compared to MVC values and reported as a percentage. Data analysis was performed using SPSS/PASW Statistics version 22.0 (Somers, NY). Means and standard deviations were calculated for the EMG activity of the $\mathrm{LD}, \mathrm{PD}, \mathrm{MT}$, and $\mathrm{BB}$ while performing each repetition of each exercise. Repeated measures analysis of variance (ANOVA) followed up with paired T-tests was used to determine if the normalized (\%MVC) EMG values for each muscle group were significantly different across the three exercises. Bonferroni adjusted p-value was applied to reduce the chances of obtaining a type I error when multiple pairwise tests were performed, which resulted in an adjusted alpha level for significance of $p<$ 0.0167. A Cohen's $d$ statistic was calculated as the effect size of the differences in \%MVC values (Cohen, 1988) and a Hopkin's scale of magnitude was used where an effect size of $0-0.2$ was considered trivial, $0.2-0.6$ small, $0.6-1.2$ moderate, $1.2-2.0$ large, and >2.0 was very large (Hopkins et al., 2009).

\section{Results}

All subjects completed the pull-up variations successfully and all data was included in the data analysis process. Means $( \pm$ SD) for $\%$ MVC values of the selected superficial musculature across the three pull-up variations are shown in Table 1 (\%MVC).

For the LD, no significant differences existed between any of the variations. The Hopkins scale of magnitude determined that effect sizes were either small or trivial for the LD between the PU variations. For the $\mathrm{BB}$, no significant differences existed between the exercises, while all effect sizes between the exercises were determined to be trivial or small. For the MT, the TP was significantly lower than the PU. Additionally, there were no differences between the SP and either the TP or PU. All effect sizes were determined to be small for the MT. Lastly, there were no significant differences between any of the exercises for the PD. The Hopkins scale of magnitude showed only small effects between the exercises.

In regards to the subjective assessment, it was determined by the subjects that the TP was the most difficult and the PU was the least difficult of the variations.

Figure 1.

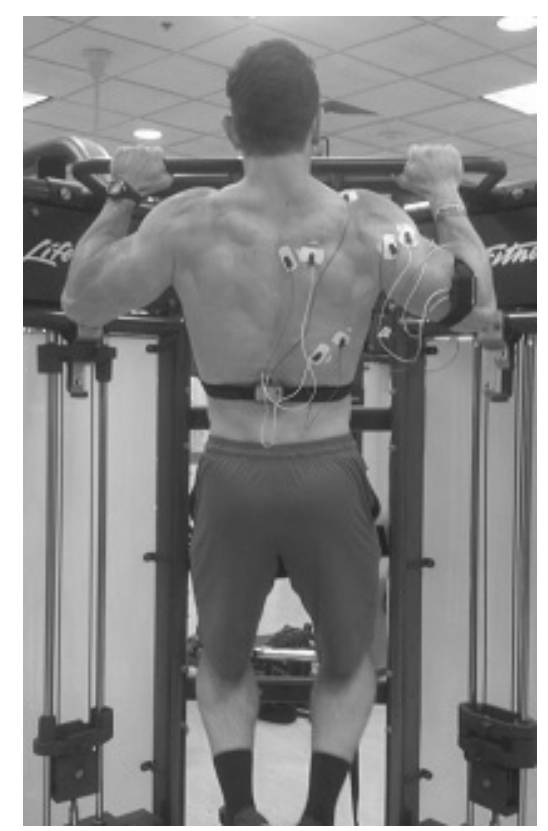

Pull-up (PU) 


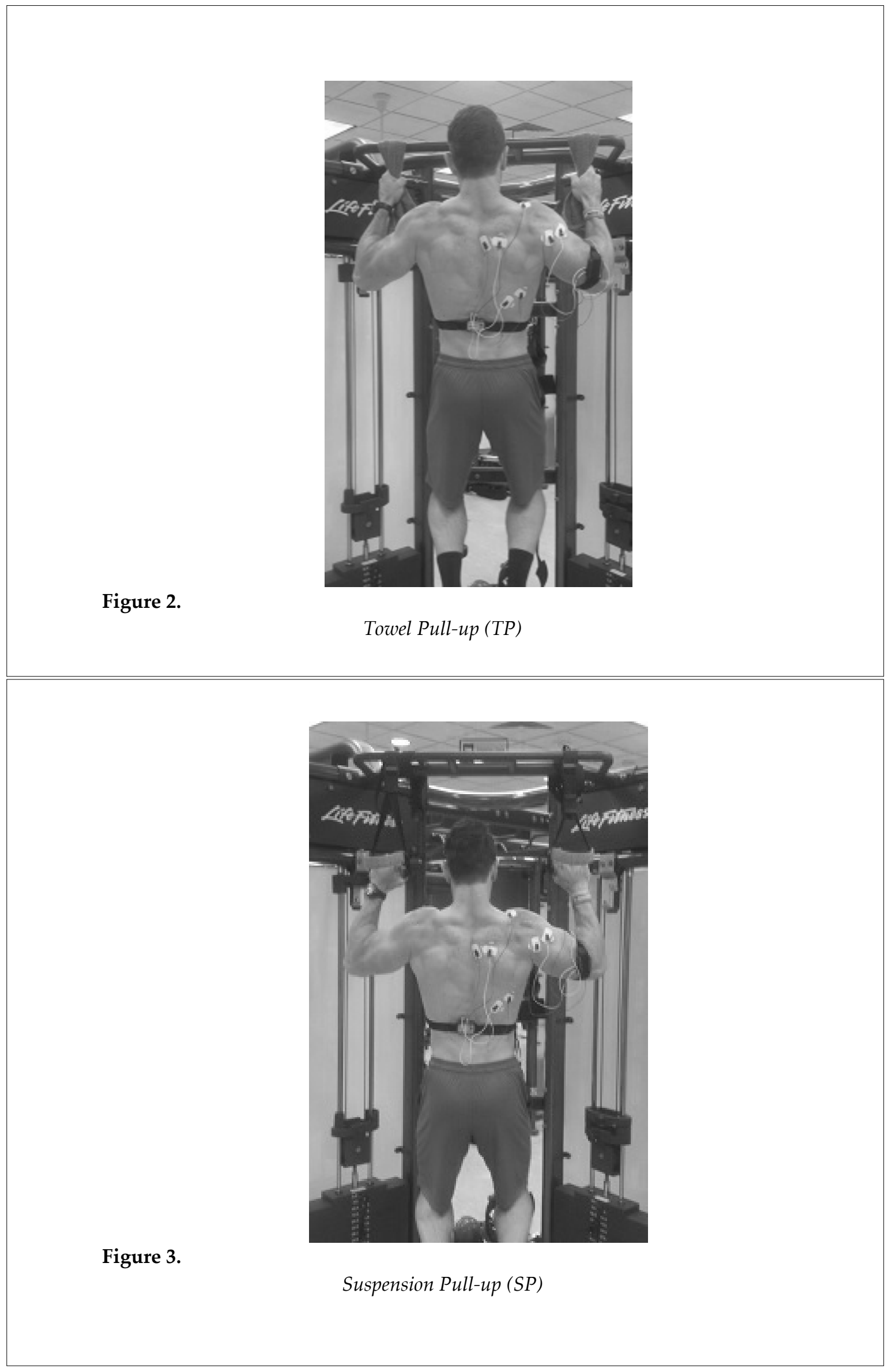

(c) EditorialCommittee of Journal of Human Kinetics 


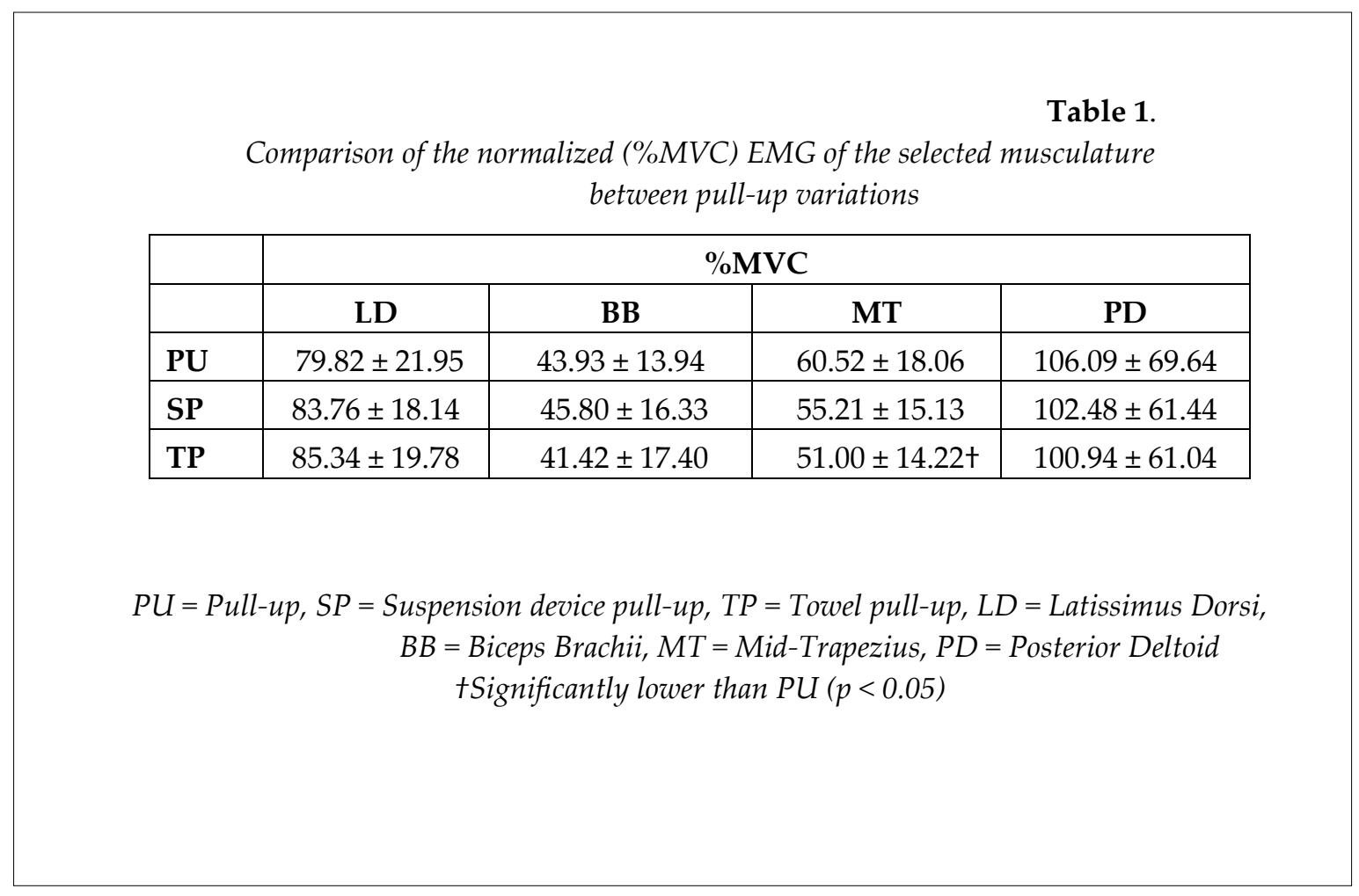

\section{Discussion}

The purpose of this investigation was to compare the electromyographical activity of the $\mathrm{LD}, \mathrm{PD}, \mathrm{MT}$, and $\mathrm{BB}$ between the PU, SP, and TP. The main finding was that only one significant difference existed (i.e., MT). Previous studies comparing resistance and therapeutic exercises have determined a base threshold of 40-60 \%MVC to invoke a sufficient stimulus to strengthen and promote muscle growth (Andersen et al., 2006; Ayotte et al., 2007). Thus, all variations examined throughout this investigation provided sufficient muscular activation levels within all muscles tested.

The findings of the current study are consistent with previous suspension device literature, performed by Snarr and Esco (2013), which compared a different pulling movement (i.e., the inverted row) on both a stable and unstable device. The study showed that while keeping grip-width constant during both types of inverted rows, the muscle activity of the LD, PD, and MT showed no significant differences. The current results continue to demonstrate that performing pulling movements with a suspension device do not appear to elicit significant increases in muscular demand.
The findings of the current study are also consistent with a previous study by Youdas et al. (2010) that compared a traditional PU and a pullup performed with the use of a Perfect-Pullup ${ }^{\mathrm{TM}}$ device. The study sought to determine the differences in EMG activity between a traditional PU and a pull-up on a device that incorporates twisting handles allowing the arms to inwardly rotate during the movement. This inward rotation, anecdotally, allows for increased muscular activity due to its' ability to mimic the natural motion of the glenohumeral joint. While no pull-ups in the current study incorporated limb rotation, studies investigating other PU devices (e.g., Perfect-Pullup ${ }^{\mathrm{TM}}$ ) also indicated no differences amongst agonistic muscle groups (Youdas et al., 2010).

During the examination of the three variations, it was determined that no significant differences occurred in LD activation. During a pull-up exercise, the LD is responsible for extension, adduction, and horizontal abduction around the glenohumeral joint (Floyd, 2009; Lehman et al., 2004). Since each variation was held at a constant grip-width, speed, and bodyweight, no EMG differences in the LD were expected. This was consistent with the author's 
hypothesis.

Additionally, no differences in the current study existed in the BB or PD between any of the exercises examined. The $\mathrm{BB}$ is an agonist during humeroulnar flexion and radioulnar supination, while the PD is responsible for horizontal abduction of the glenohumeral joint (Floyd, 2009). Though no multi-dimensional camera analysis was used, authors assumed that all three PU's provided the same degree of elbow flexion and glenohumeral joint movement since the width of hand placement and technique (i.e., chin reaching the level of the hands) were similar. Additionally, none of the PU's involved supination, thus no significant differences in BB or PD EMG activity was expected.

While not a prime mover of the pull-up, the MT was the only muscle examined to show a significant difference between the PU variations. During pulling movements, the MT is primarily responsible for scapulothoracic stabilization and retraction of the scapula (Lehman et al., 2004). The results demonstrated a significantly lower activation of the MT during the TP compared to the PU. This difference in MT activation may be caused by the differences between the movements themselves. During the PU, the subject is forced to angle the trunk backwards in order to avoid the bar during the concentric phase. This, in turn, causes additional tilting and protraction of the scapulothoracic joint; thus, additional muscle activation is required in an attempt to stabilize and retract the scapulae during the movement (Lehman et al., 2004).

The results of the current study provide insight into the muscular activation during three common pull-up exercise variations. Balanced training of the shoulder joint complex is crucial for increasing endurance and strength of the multitude of muscles responsible for glenohumeral movement (Lehman et al., 2004; Lusk et al., 2010). Traditional weight lifting programs often demonstrate a strong focus on pressing exercises with less concern for pulling movements; thereby creating a common muscular imbalance within the shoulder complex. Although a push:pull ratio for the upper body should be approximately 1.00 (Beeler et al., 2013), research has often reported significantly greater pushing values as compared to pulling (Beeler et al., 2013; Negrete et al., 2013; Schmitz et al., 2014). For example, Schmitz et al. (2014) observed significantly greater pushing strength (i.e., $10.3 \pm$ $16.3 \%)$ in college wrestlers when compared to pulling strength. Additionally, Negrete et al. (2013) demonstrated push:pull ratios ranging from 1.5-2.7 in recreationally active adults further demonstrating the increased focus on pushing exercises versus pulling. Therefore, practitioners should recognize not only the imbalances in shoulder complex musculature, but also the imbalance in strength and conditioning or rehabilitation programming that places lower importance on the posterior chain and pulling musculature.

However, this study is not without limitations. It is important to recognize that advanced techniques, such as linear encoding or video analysis, were not used during this investigation. Therefore, the breakdown of concentric and eccentric phases was not analyzed. Future research should examine the EMG activity of the primary movers of the PU with a focus on the differences during the concentric and eccentric actions. While previous research had demonstrated that instability devices cause a decrease in force output, repetitions to fatigue were not analyzed during this investigation. Therefore, the ability of an individual to perform various amounts of repetitions with one variation over another may be observed and thus, should be investigated in the future.

This multi-joint, closed-chain exercise (i.e. PU) involves movements of the shoulder, scapulothoracic and elbow joints. While the LD is a major muscle of the posterior upper torso involved in glenohumeral adduction, extension, internal rotation, and stabilization, an increased mobility of the GH joint comes at a cost of stability. Therefore, exercises, such as the PU, should be implemented into resistance training programs to enhance the strength, and perhaps, stability of the shoulder complex. While only a minor difference existed amongst the PU and TP (i.e., MT); based upon \%MVC values alone, each PU variation elicited activity above the EMG threshold required to elicit adaptations in muscular strength and hypertrophy. Thus, practitioners can be confident during programming that performing any of the PU variations examined within this study may provide significant benefits to the client or athlete. 


\section{References}

Andersen LL, Magnusson SP, Nielsen M, Haleem J, Poulsen K, Aagaard P. Neuromuscular activation in conventional therapeutic exercises and heavy resistance exercises: implications for rehabilitation. Phys Ther, 2006; 86: 683-697

Ayotte NW, Stetts DM, Keenan G, Greenway EH. Electromyographical analysis of selected lower extremity muscles during 5 unilateral weight-bearing exercises. J Orthop Sports Phys Ther, 2007; 37(2): 48-55

Beeler BA, Schmitz CD, Mayhew JL. Comparison of pushing and pulling shoulder strengths in college wrestlers. Mo J Health, Phys Ed, Recreation, and Dance, 2013; 23: 57-65.

Cohen J. Statistical power analysis for the behavioral sciences. (2nd ed.). Hillsdale, NJ: Lawrence Earlbaum Associates; 1998

Cram JR, Kasman GS. Introduction to Surface Electromyography. Gaithersburg, MD: Aspen Publishers, Inc.; 1998

Floyd RT. Manual of Structural Kinesiology. (17th ed.). New York, NY: McGraw-Hill; 2009

Harrison JS, Schoenfeld B, Schoenfeld ML. Applications of kettlebells in exercise program design. Strength Cond J, 2011; 33: 86-89

Hopkins W, Marshall S, Batterham A, Hanin J. Progressive statistics for studies in sports medicine and exercise science. Med Sci Sports Exerc, 2009; 41(1): 3

Konrad P. The ABC of EMG: A Practical Introduction to Kinesiological Electromyography. Noraxon Inc., USA; 2005

Lehman GJ, Buchan DD, Lundy A, Myers N, Nalborczyk A. Variations in muscle activation levels during traditional latissimus dorsi weight training exercises: an experimental study. Dynamic Med, 2004; 3: 4

Lusk SJ, Hale BD, Russell DM. Grip width and forearm orientation effects on muscle activity during the lat pull-down. J Strength Cond Res, 2010; 24: 1895-1900

McGill SM, Cannon J, Anderson JT. Analysis of pushing exercises: Muscle activity and spine load while contrasting techniques on stable surfaces with a labile suspension strap training system. J Strength Cond Res, 2014; 28: 105-116

Negrete RJ, Hanney WJ, Pabian P, Kolber MJ. Upper body push and pull strength ratio in recreationally active adults. Int J Sports Phys Ther, 2013; 8(2): 138-144

Ronai P, Scibek E. The Pull-up. Strength Cond J, 2014; 36(3): 88-90

Schmitz CD, Eagan BA, Arabas JL, Mayhew JL. Muscle strength imbalance at the shoulder in college wrestlers. Mo J Health, Phys Ed, Recreation, and Dance, 2014; 24: 22-30

Signorile JF, Zink AJ, Szwed SP. A comparative electromyographical investigation of muscle utilization patterns using various hand positions during the lat pull-down. J Strength Cond Res, 2002; 16(4): 539546

Snarr RL, Esco MR. Electromyographic comparison of traditional and suspension push-ups. J Hum Kinet, 2013; 39: 75-83

Snarr RL, Esco MR. Comparison of electromyographic activity when performing an inverted row with and without a suspension device. J Exerc Physiol Online, 2013;16(6): 51-58

Snyder BJ, Leech JR. Voluntary increase in latissimus dorsi muscle activity during the lat pull-down following expert instruction. J Strength Cond Res, 2009; 23(8): 2204-2209

Sperandei A, Barros MAP, Silveira-Junior PCS, Oliveira CG. Electromyographic analysis of three different types of lat pull-down. J Strength Cond Res, 2009; 23(7): 2033-2038

Willardson J. The effectiveness of resistance exercises performed on unstable equipment. Strength Cond J, 2004; 26: 70-74 
Youdas JA, Amundson CL, Cicero KS, Hahn JJ, Harezlak DT, Hollman JH. Surface electromyographic activation patterns and elbow joint motion during a pull-up, chin-up, or Perfect-Pullup ${ }^{\mathrm{TM}}$ rotational exercise. J Strength Cond Res, 2010; 24(12): 3404-3414

\section{Corresponding author:}

\section{Ronald L. Snarr}

College of Health \& Kinesiology

Georgia Southern University

P.O. Box 8076

Statesboro, GA 30460

(610) 299-6722

rsnarr@georgiasouthern.edu 